\title{
LA CRISIS DEL EURO EN PERSPECTIVA
}

Francesco Bogliacino*

Dario Guarascio**

$\mathrm{E}^{1}$ 1 proceso de integración europea ha sido tradicionalmente presentado como de naturaleza política, fundamentado en la necesidad de superar las divisiones continentales que han dejado rastros imborrables en la historia tras la mancha de las dos guerras mundiales. Efectivamente, el proyecto federal europeo tiene entre sus inspiradores el Manifiesto de Ventotene de 1941 de Altiero Spinelli, héroe en la lucha contra el fascismo (Spinelli y Rossi, 1941).

En su realización concreta, sin embargo, el proceso de integración europea $-\mathrm{O}$ sea el conjunto de instituciones comunitarias que han ido emergiendo y evolucionando- puede ser interpretado como la manifestación continental de los cambios ocurridos en el capitalismo global desde el final de la Segunda Guerra Mundial.

En la posguerra, las instituciones comunitarias -entre otras, la Comunidad Europea del Carbón y del Acero (CECA) (Tratado de París de 1951) y la Comunidad Económica Europea (CEE) (Tratado

* Doctor en Economía, profesor de la Universidad Nacional de Colombia, Bogotá, Colombia, [fbogliacino@unal.edu.co].

*** Doctor en Economía, Istituto Nazionale per l'Analisi delle Politiche Pubbliche (InApP), Roma, Italia, [dario.guarascio@sssup.it]. Agradecemos las discusiones con Federico Bassi, Valeria Cirillo, Claudio Cozza, Matteo Lucchese, Marco Missaglia, Mario Pianta, y los comentarios de Ian Seda a este escrito. Un agradecimiento particular a Christian Mongeau Ospina por sus aclaraciones sobre las series de datos BIs, y a Daniel Rodríguez por revisar la primera versión en castellano. Dario Guarascio agradece el constante y valioso debate con Giuseppe Celi, Andrea Ginzburg y Annamaria Simonazzi. Agradecemos a tres pares anónimos por sus comentarios y a David Ortiz por la revisión final. Las opiniones de Dario Guarascio son del autor y no reflejan el punto de vista de INAPP. Como siempre, los errores son responsabilidad de los autores. Fecha de recepción: 28-08-2016, fecha de modificación: 17-02-2017, fecha de aceptación: 27-04-2017. Sugerencia de citación: Bogliacino, F. y D. Guarascio, "La crisis de1 euro en perspectiva”, Revista de Economía Institucional 19, 36, 2017, pp. 257-289. DOI: https://doi.org/10.18601/01245996.v19n36.10. 
de Roma de 1957)- nacieron con la finalidad de favorecer la gestión común de las materias primas (CECA) y el intercambio comercial (y migratorio) entre los Estados miembros.

La segunda fase del proceso de integración ocurre en un contexto profundamente diferente con respecto al que acompañó la fase constitutiva de la CEE (Cafruny, 2015). El régimen de acumulación regulado por los acuerdos de Bretton Woods estaba caracterizado por una moderada competencia entre las economías, así como por el uso del dólar como moneda de referencia para los intercambios, el control administrativo sobre los movimientos internacionales de mercancías y capitales, y la prevalencia de la dimensión doméstica en la producción e inversión (Simonazzi y Ginzburg, 2015). E1 15 de agosto de 1971, Nixon declara el fin de la convertibilidad del dólar a oro, que representaba el perno de dicho sistema ${ }^{1}$.

El régimen de acumulación que siguió se puede resumir de la siguiente manera (Stockhammer, 2014; Van Apeldoorn, 2009; Celi et al., 2017): gradual liberalización de los movimientos de capitales, con un consecuente crecimiento de las dimensiones y del grado de integración internacional del sector financiero; modificación en el ejercicio de la política monetaria que se vuelve funcional a la estabilidad de los precios (necesaria para favorecer los flujos de capitales) y que pierde el rol de auxiliar de la política fiscal, como en el consenso keynesiano; las producciones masificadas de tipo fordista reducen su peso, partes del proceso productivo vienen tercerizadas tanto internamente como en escala internacional y crece la importancia de los servicios; empieza una fase de moderación salarial y de crecimiento de la participación de las ganancias, en parte debido a que las organizaciones sindicales, debido a un conjunto de reformas laborales, pierden gradualmente su capacidad de aumentar los sueldos o de reducir el horario de trabajo sin modificar el salario (Bogliacino y Maestri, 2014; Atkinson, 2015).

En este escenario, el proceso de integración europea recibe una aceleración y una parcial modificación en sus presupuestos. La dimensión política orientada a construir instituciones democráticas y soberanas en sectores como la política fiscal queda relegada a la integración económica y monetaria.

La institución de la Serpiente Monetaria -y luego del Sistema Monetario Europeo (sME)-, orientada a reducir el riesgo de cambio,

\footnotetext{
${ }^{1}$ No obstante, las instituciones creadas en Breton Woods -el Fondo Monetario Internacional y el Banco Mundial- sobrevivieron a la decisión de Nixon.
} 
tiene como elemento neurálgico la construcción de un ambiente más favorable a la libre circulación de mercancías y capitales ${ }^{2}$.

E1 euro constituye el sello de esta segunda fase. Una vez establecidas las condiciones para su introducción, entre las cuales se subrayan las reglas fiscales (relación déficit/Pıв del 3\%, relación deuda pública/PIв del 60\%), a través del Tratado de Maastricht (1992), la moneda única implica la renuncia definitiva a la soberanía monetaria y la irreversibilidad del vínculo externo para los países de la Unión Monetaria Europea (UME). Los primeros países en adoptar el euro en 1999 son: Alemania, Austria, Bélgica, España, Finlandia, Francia, Irlanda, Italia, Luxemburgo, Países Bajos y Portugal. Grecia adoptó la moneda única en el 2001 y le siguieron Chipre, la República Eslovaca, Eslovenia, Malta, Estonia, Letonia y Lituania. Paralelamente se introducen reglas que liberalizan por completo la circulación de ciudadanos de los Estados miembros y de las mercancías producidas en los mismos. La unificación alemana determina un desbalance del baricentro económico europeo hacia el centro, ya que se genera un proceso de divergencia entre centro y periferia, con el primero que aumenta su peso económico y la segunda que ve debilitarse su estructura productiva y aumentar su dependencia de los capitales externos (Simonazzi et al., 2013; Cirillo y Guarascio, 2015)³.

\footnotetext{
${ }^{2}$ Tanto la Serpiente Monetaria como el sme son acuerdos intergubernamentales que implican la renuncia, por parte de los adherentes, al instrumento de la tasa de cambio e implican una restricción en la capacidad de utilizar la política monetaria en función de modificar las dinámicas reales. La imposibilidad de devaluar la moneda, además, coincide con la clausura de una válvula en el caso de excesivo déficit de balanza de pagos. Este último fenómeno conduce a presiones sobre los salarios a la hora de promover un ajuste, provocando un debilitamiento del trabajo y la institución de un 'vínculo externo'. En parte la creación de la Serpiente Monetaria en 1972 nace del deseo de estabilizar las monedas (Flassbeck y Lapavitsas, 2015), sin embargo, los cambios ocurridos en los años setenta, y en particular el nuevo régimen de movimientos de capitales, hacen que el sME de 1979 ya se inscriba completamente en el nuevo orden de la globalización bajo la guía de Estados Unidos (Panitch y Gindin, 2012). En este sentido la interpretación del euro como una alternativa al dólar adolece de profundidad de análisis. Primero, frente a la negativa de Alemania a implementar políticas expansivas, es evidente que el euro puede funcionar solo en superávit externo, de lo contrario los países de la periferia no podrían nunca encontrar la demanda suficiente para crecer. Si es así, el euro no provee liquidez a nivel internacional y no podría en ningún momento remplazar al dólar. Segundo, el proyecto de integración monetaria fue favorecido por Estados Unidos, sobre todo la constitución del SME en 1979, para limitar las posturas keynesianas y aislacionistas en el Reino Unido y Francia (ver Barba y Pivetti, 2016).

${ }^{3}$ Actualmente, la Unión Europea entra en nuevos acuerdos de libre comercio con China. Este impulsa los procesos de internacionalización financiera y productiva, que a la vez refuerzan la moderación salarial, gracias a las mayores posibilidades de relocalizar (Bogliacino et al., 2016) y la posibilidad de aumentar exógenamente el salario real a través de importaciones a bajo precio (Simonazzi et al., 2013).
} 
E1 discurso político europeo, en calidad de discurso performativo y no como único medio de reproducción de lo real (Foucault, 1970), se actualiza. Si bien los Estados Unidos de Europa y el ideal europeo de Spinelli permanecen como marco de referencia dentro del cual construir consenso para las nuevas instituciones monetarias, se introducen dos cambios. En primer lugar, la remoción de las barreras de circulación se vuelve un valor en sí, porque supuestamente favorecería la convergencia hacia arriba de los ingresos (Frenkel y Rose, 1997); en segundo lugar, se formula la tesis de la reacción en cadena, según la cual la integración monetaria habría llevado a la integración fiscal y política. El discurso dominante se fundamenta, ahora, en dos visiones que, si bien se originan en perspectivas muy diferentes, terminan sustentándose. La visión idealista, en virtud de la cual el objetivo final de la Europa Federal es ineluctable e independiente de las condiciones económicas subyacentes (Negri y Sánchez, 2015); y una ideológica -de matiz neoliberal y monetarista-, basada en la idea de que la moneda común habría inducido de forma natural, vía convergencia, las condiciones óptimas para la realización de la unión fiscal y política (Sinn, 2014; Frenkel y Rose, 1997). Las consecuencias de la primera visión son particularmente negativas: el imaginar que la política lleve el timón sin restricciones responde a un enfoque ingenuo, que no considera que el margen de acción político en Europa está directamente determinado por el sistema de instituciones económicas (el famoso piloto automático, denunciado por Krugman en 1998), y que no es posible que decisiones de policy puedan corregir el camino cuando operan en un contexto institucional disfuncional.

El entrelazado de estas dos visiones ha legitimado ex post el diseño de las instituciones europeas y ha representado la base técnicoideológica para el conjunto de políticas de austeridad en la poscrisis. Ambas visiones parecen sufrir de una aporía fundamental ${ }^{4}$. Por naturaleza, se olvidan de los elementos de heterogeneidad entre estructuras productivas, así como del papel del conflicto distributivo en la determinación de las dinámicas económicas y los sesgos de los sectores financieros en la asignación de los recursos. Estas falacias han impedido difundir la consciencia de las divergencias y la inestabilidad que se iban acumulando en los acontecimientos precedentes a la crisis, jugando un papel clave en el estallido de la misma (Lapavitsas, 2012).

${ }^{4}$ Una visión similar es la de Cafruny (2015) que ve en la UME la culminación de un proceso que requiere la unificación política para preservar la unificación monetaria, y señala como ejemplo la trayectoria desde el Plan Werner hasta Maastricht. El valor añadido de nuestro artículo es discutir el elemento del discurso dentro de las dinámicas económicas que conducen a la inestabilidad del proyecto. 
La gravedad de esta falacia institucional e interpretativa se ha mostrado en toda su claridad desde que la Unión Europea ha empezado a enfrentarse a las crisis, primero financiera, luego exterior (por el tema de los refugiados), finalmente política (por la salida del Reino Unido), y en un futuro cercano es muy posible que de nuevo sea financiera (por el tema de la cartera morosa).

E1 objetivo de este artículo es discutir desde una perspectiva de análisis económico e institucional los límites y los sesgos implícitos en el proceso de integración europea considerando en particular el caso de la UME. Adicionalmente, se muestran los límites de los discursos de la integración europea (el idealista-político y el ideológico-económico) evidenciando la conexión entre ellos, el diseño de la UME y las políticas de austeridad adoptadas en la poscrisis.

La pregunta principal de este artículo se puede formular así: ¿cómo pudo ocurrir que una entidad institucional que proponía convergencia y armonización esté llevando a una de las zonas más ricas del planeta al borde del colapso? Para intentar dar una respuesta, nuestra convicción es que hay que adoptar una perspectiva que sea: a) sistémica, esto es, que analice el proceso de integración europea dentro del cambio de paradigma internacional que lleva al abandono del keynesianismo; $y \mathrm{~b}$ ) histórica, en el sentido de mostrar las raíces de un diseño institucional que favorece la divergencia y las desigualdades. Este artículo tiene varios elementos de novedad respeto a la literatura existente sobre la crisis del euro. Primero, explica cómo la construcción monetaria europea sigue las tendencias del capitalismo mundial y por lo tanto se tiene que interpretar a la luz de dinámicas estructurales y geopolíticas. Segundo, muestra las peculiaridades históricas del proceso europeo y la dinámica centro-periferia implícita en este proceso. Tercero, analiza los discursos a favor de la integración monetaria y muestra cómo su función ha sido sobre todo la de legitimación de las políticas que respondían a los vínculos estructurales. En este sentido nos distinguimos de buena parte de la literatura que critica el euro como un proceso mal diseñado por cuestiones ideológicas (y no estructurales) y que solamente discute una de las dos posturas teóricas (la neoliberal o de convergencia), desconociendo el rol de la visión idealista (el caso más reciente es Stiglitz, 2016). Cuarto, y último, este ensayo muestra cómo la austeridad se explica por la lógica institucional, y no como una (mala) decisión arbitraria de las autoridades europeas.

Este escrito continúa de la siguiente manera: la primera sección analiza la insostenibilidad del euro como acuerdo cambiario; la segunda presenta un análisis de la respuesta a la crisis; la tercera analiza 
el caso griego; la cuarta muestra la naturaleza contradictoria de las instituciones creadas antes y después de la crisis, y la última presenta las conclusiones.

\section{RACIONALMENTE IRRACIONAL}

E1 23 de mayo de 2013, invitado por el Banco de Grecia, el vicepresidente del вCE, Vítor Constâncio, declaró:

E1 principal determinante de la crisis se localizó en el sector financiero, en particular en los bancos que intermediaron ingentes flujos de capitales hacia la periferia, creando desequilibrios que se volvieron insostenibles una vez una interrupción imprevista ocurrió en seguida de la crisis financiera y la brusca revisión del precio del riesgo que conllevó (Constâncio, 2013).

La crisis europea efectivamente llegó desde afuera ${ }^{5}$. El colapso del sistema financiero norteamericano impactó directamente al sistema europeo, en razón de la exposición a activos tóxicos, particularmente alta en el caso del sistema bancario alemán y francés, e indirectamente, vía reducción de la demanda de Estados Unidos y de Asia. Cuando los bancos del centro del sistema europeo fueron golpeados, pretendieron reducir la exposición que tenían dentro del continente, y resultó que esta exposición afectaba prevalentemente la periferia del sistema euro.

La crisis de las hipotecas basura puso en duda la sostenibilidad de la UME y de la dinámica financiera intraeuropea. Como se muestra en la gráfica 1, con datos de Eurostat, en 2009 las tasas de interés de los países de la periferia empiezan a divergir respecto a las de Alemania, a pesar de compartir la misma moneda.

Como se anticipó en la introducción, la moneda común se fundamenta en una visión de naturaleza económica que se centra en un concepto clave: la convergencia. Si los movimientos de capitales son libres, los flujos se mueven desde los países donde su rentabilidad es baja hacia países donde su rentabilidad es alta. Lo anterior beneficia a estos últimos, que típicamente son países con un menor grado de desarrollo, permitiendo una fase de crecimiento sostenido. Mientras estos países convergen (en un sentido matemáticamente preciso), se estabilizan tanto las tasas de crecimiento como las tasas de rentabilidad y los capitales se mueven hacia otro lado. E1 mundo de libre movi-

${ }^{5}$ E1 hecho de que llegue desde afuera no matiza la responsabilidad del diseño del euro: siempre una economía tiene que enfrentar crisis (más o menos graves), y la crítica al sistema euro es precisamente que no es capaz de soportar choques asimétricos a causa de su inflexibilidad. Lo mismo se podría decir por ejemplo del impacto de la crisis reciente de Nokia en Finlandia: obviamente se trata de una empresa muy grande para la cual se puede desarrollar un discurso específico de responsabilidades, pero un sistema económico tiene que ser capaz de absorber choques, independientemente de la naturaleza de los mismos. 
miento de capitales sería, en efecto, capaz de asignar eficientemente los recursos. Esta narración ha inducido a los representantes de países con estructura productiva relativamente débil o poco extensa a aceptar la adopción de una moneda sobrevaluada ${ }^{6}$.

Gráfica 1

Convergencia y divergencia de los tipos de interés



Fuente: Eurostat. Serie para el criterio de convergencia del Tratado de Maastricht. Tipos de interés a 10 años para los gobiernos.

Ya es un hecho reconocido por toda la literatura que esta visión no tiene fundamento: la convergencia entre las economías más avanzadas y las más atrasadas no se logró, sino que creció el endeudamiento y la dependencia de la periferia hacia el centro de la zona euro (Lapavitsas, 2012; Simonazzi et al., 2013). De hecho, más que a una dinámica de integración y subsiguiente convergencia, el euro contribuyó a la materialización de una relación de dependencia entre las economías del sur de Europa (receptoras de capitales y cada vez más débiles en términos de estructuras productivas y capacidad industrial) y el centro, de manera similar a lo que ocurrió entre los países de América Latina y Estados Unidos en la época del currency peg.

En 1985, Carlos Díaz-Alejandro publicó un artículo en donde hacía un balance de la historia financiera de América Latina. En los años setenta, Chile tenía un régimen dictatorial, y por lo tanto ninguna presión democrática sobre la toma de decisiones. Además el

${ }^{6}$ En el momento de la adhesión, los responsables de la política económica del Sur dieron más peso a los beneficios de los movimientos de capitales -causados por la eliminación del riesgo de cambio- que al costo de la penalización en términos de capacidad competitiva (por la sobrevaluación del cambio) y pérdida de la soberanía monetaria. 
Ministerio de Hacienda estaba rodeado de los famosos Chicago-boys, cuyo pedigree a favor del laissez faire no podía ser cuestionado.

El sector financiero, muy desregulado, tuvo grandes presiones en el periodo 1976-1977. Las autoridades -recuerda Díaz-Alejandroemitieron comunicados recordando su no rotundo a intervenciones y la reafirmación de la libertad de quiebra en todos los sectores. Al final de los setenta ocurrían dos cosas: involucramiento en los problemas de un banco grande (Banco Osorno) y un acuerdo de cambio con Estados Unidos, al cual siguió una entrada masiva de capitales; mientras tanto la prensa celebraba la atracción del sistema financiero chileno y la baja deuda pública, pero la inflación entre los dos países no mostraba ninguna convergencia inmediata.

A principios de los años ochenta, el país enfrentó las primeras intervenciones de entidades por parte del banco central, luego siguió el protocolo de siempre: el gobierno tuvo que intervenir masivamente en el mercado, socializando los activos de las entidades en dificultad e incluyendo las deudas externas; igualmente, se devaluó la moneda de manera notoria, y se generó una masiva redistribución de riqueza. Lo más importante es que las recomendaciones y las declaraciones iniciales fueron completamente desatendidas (Díaz-Alejandro, 1985).

Para quien conozca los hechos de la zona euro, dicha secuencia parece bastante llamativa. Este punto lo subraya Frenkel (2014): desde la ola de crisis latinoamericanas de los ochenta, a la del Sureste asiático de final de siglo, a la del euro, el patrón común es un ciclo de boom financiero y una caída repentina, pasando por una fase intermedia de burbuja y de alta inflación y crecimiento. En detalle, se empieza con una política de liberalización financiera acompañada por una serie de acuerdos que limitan la flexibilidad en el tipo de cambio. Los capitales que entran masivamente no muestran capacidad de seleccionar de manera apropiada el perfil de confiabilidad de los deudores, y se generan burbujas en activos.

En la fase creciente del ciclo de Frenkel hay una aceleración del crecimiento, de la tasa de inflación para bienes no transables, y de la deuda privada y externa. El detonante de la crisis es un choque externo, que cambia las percepciones de los agentes en el mercado. Muchos cierran posiciones y buscan liquidez, se desinflan las burbujas y los casos de iliquidez e insolvencia se multiplican, llevando a la crisis ${ }^{7}$.

\footnotetext{
${ }^{7}$ Como el mismo Frenkel (2014) afirma, detrás de este movimiento está la hipótesis de inestabilidad financiera de Minsky (1975), según la cual las expectativas del sistema financiero se inflan y desinflan, generando secuencias de burbuja y estallidos. Detrás de este escenario macro hay un argumento microeconómico lógicamente poderoso: no es racional hacer previsiones basadas en los "fundamentales"
} 
La coherencia de los hechos estilizados con el ciclo de Frenkel es evidente. En el cuadro 1 se presenta un abanico de indicadores financieros. Como se puede ver, no hay variaciones de la deuda pública que justifiquen el patrón de desarrollo de la crisis. Los países de la periferia tenían niveles de deuda tanto por encima (Grecia e Italia) como por debajo (Irlanda y España) de Alemania. La variación tampoco explica mucho: en Irlanda, España e Italia la variación en relación al PIв es negativa; en Grecia es positiva pero comparable a cuanto ocurre en Francia y Alemania. El único caso de incremento significativo sería Portugal, donde hay una convergencia hacia los valores de Francia y Alemania, mostrándose un incremento de alrededor de 17 puntos.

Lo que se mueve de manera coordinada en todos los países es la deuda privada, que crece en 30 puntos del рів en Italia (del 71,8\% al 109,7\%), en 60 en Grecia (del 44,1\% al 101,9\%), en Portugal del $124,5 \%$ al $185 \%$, en Irlanda del 139,4\% al 197,8\%, y en casi 100 puntos en España (del 93,6\% al 191,2\%). Lo mismo ocurre con la deuda del sector financiero. La deuda privada es financiada de manera consistente por el exterior en todos los países de la periferia, cuya posición neta externa empeora.

Mientras sigue la euforia, la entrada de capitales infla burbujas que generan crecimiento e inflación (el diferencial acumulado de inflación entre centro y periferia es en promedio de 15 puntos). La periferia ve deteriorarse la balanza de pagos: en un primer tiempo, los capitales compensan y las reservas suben (Frenkel, 2013); pero con las primeras amenazas de sostenibilidad, hasta la inversión extranjera directa (IED) termina afectando negativamente la posición de la cuenta corriente, vía exportaciones de las ganancias.

De acuerdo con el esquema Minsky-Frenkel, en la crisis se presentan las siguientes fases: un periodo de convergencia de las tasas de interés, que sigue a la introducción del euro y al fin del riesgo de cambio (1999-2000); la entrada de capitales del centro a la periferia (2001-2008); la interrupción abrupta de los flujos de capitales y el comienzo del desapalancamiento, que coincide con la divergencia de las tasas de interés (2008-2009); el fin del ciclo financiero, con el estallido de la crisis y la recesión, y la gradual pero incompleta rebaja de

cuando el mercado muestra grupos de agentes con expectativas "correlacionadas". Pensar que agentes racionales no harían transacciones comprando por encima del precio de equilibrio cuando sepan que encontraran otros dispuestos a comprar aún más caro, es imaginar que el equilibrio prima sobre el desequilibrio y negar el presupuesto de racionalidad que se quiere afirmar (Fisher, 1983). Es lo que afirma Keynes en el capítulo 12 de la Teoría general y lo que muestra de manera rigurosa la moderna teoría comportamental de las finanzas (De Long et al., 1990). 


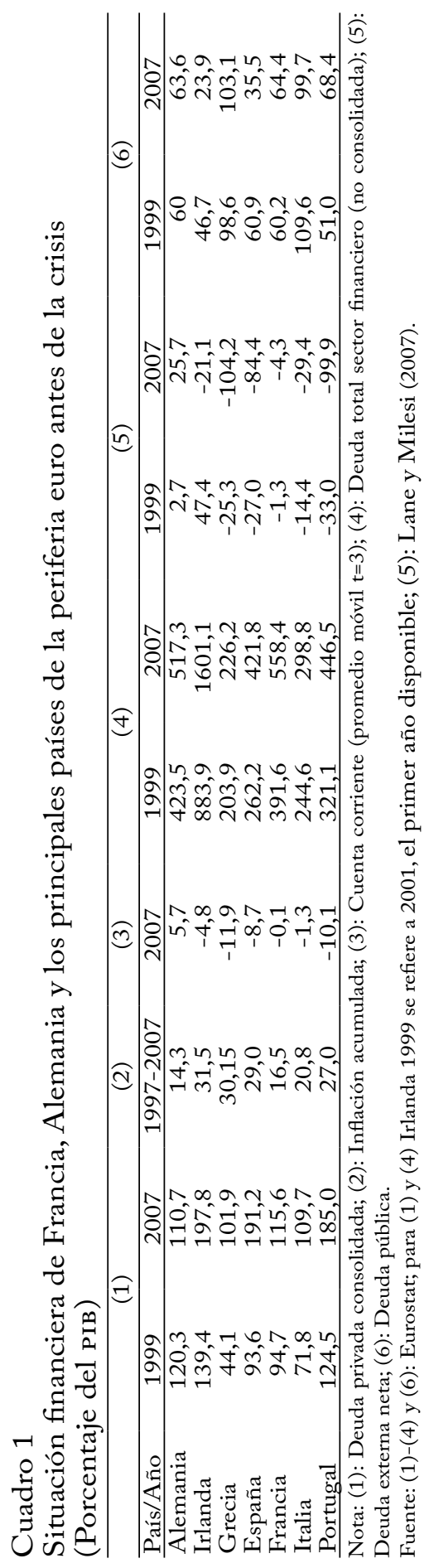

Revista de Economía Institucional, vol. i9, N.o 36 , primer semestre/2oi7, pp. $257^{-289}$ 
las tasas de interés en todos los países, menos en Grecia, determinada por la intervención masiva del вСЕ (O'Connell, 2015).

Es preciso identificar dos factores financieros clave. El primero es el endeudamiento orientado a la compra de bienes extranjeros, desde el sector privado de la periferia hacia el sistema financiero del centro. En este caso el papel central de Alemania y su red productiva es crucial, como se puede apreciar en la gráfica 2, donde reportamos el saldo comercial bilateral entre los países de la periferia del euro y Alemania. Estos flujos son sostenidos por la amplia disponibilidad de crédito, determinada, a su vez, por la necesidad de los intermediarios financieros del centro de buscar destinos para el exceso de ahorro asociado al excedente externo.

Gráfica 2

Saldos comerciales bilaterales: millones de dólares, precios corrientes

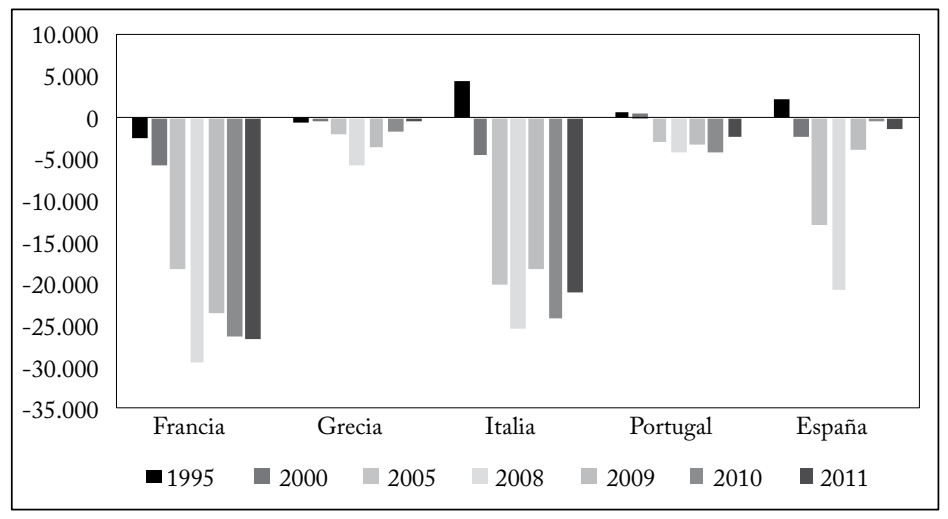

Fuente: TIVA-OECD, elaboración de los autores.

El segundo factor tiene que ver con las burbujas financieras que han afectado al sector construcción de los países de la periferia, como se evidencia por el índice del precio de la vivienda que se reporta en la gráfica 3, donde se ve cómo desde 2006 hasta 2009 el incremento de los precios de la vivienda en España (donde la burbuja ha sido más fuerte) e Italia (donde el fenómeno ha sido más contenido) es sistemáticamente superior al de Alemania. Desde 2009 se genera un desplome de esos precios en los dos países, mientras que Alemania vive el fenómeno opuesto.

Las bajas tasas de interés y el exceso de liquidez favorecen en la periferia las iniciativas del sector de la construcción. El segundo factor, en particular, induce a rebajar los requerimientos para la concesión de préstamos, creando un efecto demanda que empuja el precio de la 
vivienda, con efectos positivos sobre los balances de los intermediarios financieros, que han inscrito esos inmuebles entre las garantías. Esto favorece el incremento de inversiones por el mecanismo antes explicado. E1 ciclo se interrumpe por la llegada de un choque externo o de un momento de incertidumbre. El crédito y la demanda se contraen, las expectativas sobre el rembolso de los préstamos empeoran, se desploman los precios y se devalúan los balances de los bancos. A nivel macro, se genera un impacto negativo sobre el crecimiento; a nivel micro, empieza una serie de quiebras en el sector inmobiliario, de la construcción y, en casos más graves, en el financiero.

Gráfica 3

Índice del precio de la vivienda, varios países

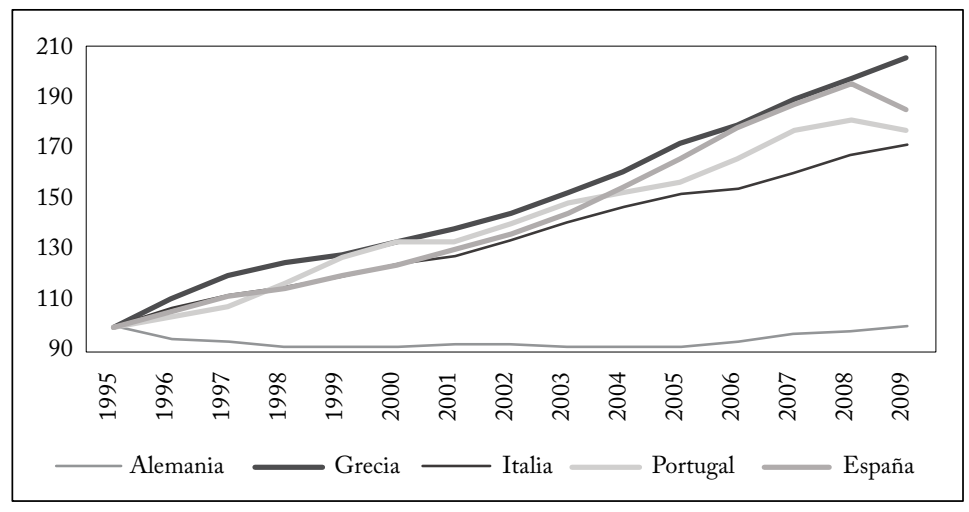

Fuente: deflactor del sector inmobiliario: wiod (1995=100), elaboración de los autores.

Los potenciales de inestabilidad no eran algo inesperado en la época: toda la academia de Estados Unidos y la Reserva Federal argumentaban en contra de la sobrevivencia del euro (Jonung y Drea, 2010). La objeción norteamericana tenía que ver con que los países que iban a ingresar a la nueva moneda europea no cumplían con los requerimientos de un área monetaria óptima (AMO) (Bagnai, 2012a). ¿Qué es una AMo? La literatura trata el problema como una cuestión de costos y beneficios: por un lado, la ganancia de un mercado común con mayor integración; por el otro, los costos de los ajustes. Este último punto fue la intuición original de Mundell (1961). Una Amo es un sistema económico que sería capaz de absorber los choques externos asimétricos, en ausencia del instrumento de ajuste operado por la tasa de cambio.

Si una región es golpeada por un choque, se necesita un mecanismo de ajuste. Las condiciones que permiten mantener la misma moneda 
son, entonces: un ciclo de negocio homogéneo entre sus regiones (que eliminaría de raíz la necesidad de políticas monetarias diferentes), alta movilidad de trabajo, precios y salarios flexibles y un mecanismo fiscal de compensación. Es obvio que Europa -donde la soberanía recae en manos de los diferentes países, y existen diferencias culturales y de estructuras económicas, así como inflexibilidades salariales por el sistema de bienestar y protección- no cumplía con ningún criterio. La defensa europea era que, políticamente, se podía generar de manera endógena un área óptima.

En la literatura económica existía un trabajo de Frankel y Rose (1997) que argumentaba de la siguiente manera: si la moneda común permite mayor integración respecto a las condiciones iniciales, el ciclo económico se coordinará, las ganancias aumentarán y los criterios para la AMO se ajustarán de manera endógena (Frankel y Rose, 1997; Jonung y Drea, 2010).

Ex post, los datos aclararon quién tenía la razón ${ }^{8}$. Como se mostró en esta sección, se puede encontrar una similitud entre la inestabilidad del área euro y las dinámicas América Latina-Estados Unidos de los años ochenta. Además se argumentó que la visión ideológicoeconómica soportó el diseño institucional del euro. En la próxima sección se muestra cómo los desequilibrios financieros privados que detonaron la crisis guiaron la selección de la senda de política económica para enfrentarla. Sin embargo, un elemento que jugó un papel clave fue la visión idealista-política de la Unión Federal, que movilizó un argumento tipo TINA (there is no alternative) generando el consenso necesario para el proceso político.

\section{LA CRISIS Y LA AUSTERIDAD}

Las presiones sobre el sistema financiero de estos países durante los años de la crisis obligan a fuertes intervenciones de los gobiernos, socializando las pérdidas del sistema bancario. La historia de Chile, Corea o (más recientemente) Estados Unidos se repite. Además, muchos de estos países tienen estabilizadores automáticos, como los subsidios de desempleo ${ }^{9}$, que afectan el gasto, y la recesión frena el recaudo tributario.

\footnotetext{
8 Por supuesto, "tener la razón" significa identificar al menos un riesgo de sostenibilidad. E1 hecho de que el euro haya sido salvado por lo menos dos veces implica que existía un riesgo. Así como en el caso de los terremotos solo podemos prever riesgos sísmicos, y no los eventos puntuales, aquí solo se pueden identificar áreas de inestabilidad, y no las crisis financieras.

9 Paradójicamente, el actual proceso de austeridad y recorte hizo que estas políticas de estabilización fueran infructuosas (Stiglitz, 2016).
} 
Por lo tanto el déficit crece. En 2009, Irlanda tiene un saldo neto del $-13,8 \%$ del PIв, que se dispara hasta el $-32,3 \%$ el año siguiente, cuando habrá que salvar los bancos. El déficit de Grecia en los dos años considerados es del 15,2\% y el 11,2\% del PIB; en España del 11\% y el 9,4\%; en Italia del 5,3\% y el 4,2\%, y en Portugal del 9,8\% y el 11,2\%. Francia, anticipándose a lo que vendrá enseguida, llega a un déficit del 7,2\% y el 6,8\%. Alemania lo empeora, del 3,2 al 4,2\%.

La sostenibilidad del sistema financiero y el hecho de que la responsabilidad última de las deudas impagas recae sobre el gobierno disparan la alarma sobre la sostenibilidad del euro. Sube el spread, lo que genera una espiral de mayor pago de intereses, mayor déficit y mayor deuda pública.

Varias anotaciones son importantes. Primero, la exposición financiera hacia la periferia es principalmente un asunto de bancos alemanes y franceses. En Grecia, por ejemplo, el вNP Paribas y Commerzbank eran los más expuestos (Morris, 2012). Sin embargo, el tamaño del sector financiero y de la deuda griega era minúsculo frente a los activos totales del centro. Como reportan Ardagna y Caselli (2012), con datos del Bank of International Settlements, la exposición alemana equivalía al $0,3 \%$ de los activos y la francesa al 0,55\%. Lo que podía asustar era un efecto contagio. La quiebra de Lehman Brothers, el 15 de septiembre de 2008, estuvo a un paso de arrastrar a todo el sistema financiero mundial, haciendo desaparecer el mercado interbancario. El miedo en este caso era el de un efecto contagio: ¿qué pasaría si cayeran Italia, España, Irlanda y Portugal ${ }^{10}$ ?

En momentos críticos, el discurso político juega un rol clave. Por un lado, la visión idealista lleva a agregar todo el consenso y a minusvalorar los sacrificios de las clases populares en nombre del ideal de integración. Por otro lado, no faltan tonos y matices morales en contra de los países de la periferia del área euro, como si detrás de un deudor en problemas no estuviera un acreedor que no hizo bien sus evaluaciones (D'Angelillo y Paggi, 2012).

La academia jugó también un papel importante: en esa época propone la tesis de la austeridad expansiva. Alesina y Ardagna (2009) ponen en duda la idea de que la austeridad del sector público afecta necesariamente y de forma negativa el crecimiento. En 2010 Ameri-

${ }^{10}$ Se puede notar cómo se cumple sistemáticamente la afirmación de Stiglitz (2002) según la cual las reglas del sistema financiero en la globalización han contravenido la supuesta lógica del capitalismo, según la cual cada uno es responsable de sus inversiones. Cuando los errores tocan los bancos, las inversiones se recuperan con la intervenciones del Estado, bajo el argumento (ex post incontestable) de que ciertos bancos son demasiado grandes para quebrarse. 
can Economic Review publicó un artículo de Reinhard y Rogoff en el que afirman que, cuando la deuda pública supera el 60\% del PIB, el crecimiento se reduce históricamente dos puntos, y si supera el $90 \%$ el crecimiento se reduce a la mitad. Tal vez fue, únicamente, una legitimación científica para decisiones que se habrían tomado de todos modos, pero funcionó. La historia nos cuenta que el primer artículo identifica la austeridad a través de la evolución del saldo del balance público, endógeno respeto al crecimiento, y el segundo en realidad estaba basado en un error en la fórmula utilizada en la hoja de cálculo (Herndon et al., 2014).

"Austeridad" es el término que indica un ajuste fiscal a través de mayores impuestos o recortes al gasto público. Su objetivo declarado es el ajuste del déficit público primario (excluyendo el gasto por intereses) como proporción del PIB, y su objetivo oculto es la corrección del déficit externo (balanza de pagos).

El ajuste de alícuotas y rubros de gastos afecta tanto positiva como negativamente el objetivo declarado: reduce el gasto, pero recorta la demanda interna afectando negativamente el pIв (que es el denominador de la variable objetivo) e indirectamente el mismo recaudo fiscal, vía base gravable. Son dos efectos que van en sentido contrario, sin embargo, como admite el mismo FMI (Blanchard y Leigh, 2013), ya que los efectos multiplicadores son mayores que uno, el efecto indirecto termina siendo dominante.

Por otro lado, la austeridad del Estado reduce la demanda interna y por lo tanto también la demanda por bienes importados, y, al crear más desempleo, disciplina a los trabajadores y baja los sueldos, incrementando la competitividad externa. Dos efectos dolorosos, pero eficaces para reequilibrar el déficit externo. Al final de cuentas, la austeridad fracasa en su objetivo explícito, pero funciona en su objetivo verdadero.

En 2011 el primer ministro de Irlanda, Brian Cowen, pide la intervención de la UE y del FMI y renuncia al cargo, lo que después de una elección llevaría al poder al Fine Gael, en coalición con el Labour.

El mismo año, en Portugal, el gobierno de centro-izquierda (sin mayoría) intenta pasar un ajuste fiscal impuesto por la UE a cambio de un rescate. La oposición pide un voto en el Congreso y, frente a la derrota, el primer ministro Socrates renuncia. E1 5 de abril el Presidente anuncia elecciones para el 5 de junio de 2011; sin embargo, el 6 de abril se pide formalmente el rescate. Las elecciones serán ganadas por la derecha de Passos Coelho que de todos modos se había comprometido a respetar las condiciones del rescate. 
En Italia, el primer ministro era Silvio Berlusconi, conocido por sus excesos e intemperancias y por su complicada relación con la justicia. Durante todo el año 2011 crece el diferencial con las tasas de interés de Alemania. En agosto de 2011, el все escribe una carta al gobierno italiano, con detalladas medidas que se deben implementar para poder recibir su apoyo y calmar a los mercados comprando títulos italianos en el mercado secundario. En noviembre, el presidente de la República nombra senador vitalicio a Mario Monti, tecnócrata cercano a las instituciones europeas, y le confiere el encargo de nombrar un gobierno técnico, después de la dimisión de Berlusconi.

En Grecia, el pAsoK (socialista) es elegido en 2009 y el primer rescate financiado por la troika es pactado en 2010. En 2011, el primer ministro decide dar un paso al lado para permitir la posesión de un gobierno técnico, apoyado por una gran coalición según el modelo alemán, en manos del ex vicepresidente del все Lucas Papademos.

Estos hechos muestran en la práctica el piloto automático.

Hay ciertos elementos técnicos de la arquitectura monetaria europea que son importantes. Primero, el sistema europeo de los bancos centrales mantiene el control de la política monetaria en Frankfurt y eso impide flexibilidad interna para abordar choques asimétricos. Segundo, hay libre circulación de capitales y en cuanto tal, libre "salida" de capitales ${ }^{11}$. Tercero, el banco central no puede acudir al mercado primario de emisión de nuevos títulos por parte del gobierno (Tratado de Maastricht, art. 104). Esto implica que los precios de emisión de títulos son determinados por parte del mercado secundario, ya que Hacienda no puede garantizar rentabilidad menor a la prevalente en dicho mercado. En este sentido se explica el "juicio" de los mercados sobre los gobiernos. Cabe recordar que en los periodos de máxima tensión para Grecia e Italia, el Financial Times reportó que se habían abierto las ventanas para cerrar posiciones cortas en el mercado secundario, subiendo la presión especulativa (Kaminska, 2011; Hope, 2010; de Leo, 2012). Cuarto, el sistema bancario tiene una línea de acceso al все para tener liquidez (emergency liquidity assistance, ela). Esta última es completamente discrecional.

${ }^{11}$ Este elemento es mitigado parcialmente por la existencia de un sistema de pagos infra-europeo (TARGET 2) que permite el funcionamiento regular de la eurozona. Cada vez que se realiza una transacción entre dos contrapartes de dos países, el cierre se da a través de un mecanismo de clearing que involucra los bancos centrales y el Eurosistema en conjunto. No hay límites al uso de TARget 2, de lo contrario el sistema de pagos se bloquearía. Esto permitió financiar la salida de capitales en el momento más duro, sobre todo en Italia y España (Higgins y Klitgaard, 2014). 
Existe un marco financiero-monetario que controla los espacios políticos nacionales (como una pistola apuntada), que va más allá de la condicionalidad de los rescates.

Gráfica 4

Saldos sectoriales y deuda pública: Grecia 2007-2013

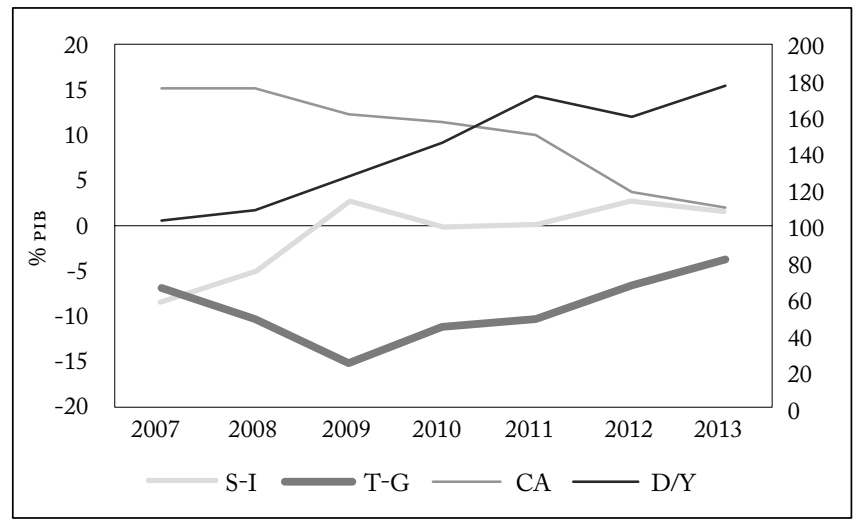

S-I: ahorro neto privado, T-G: ahorro público neto, CA: déficit en cuenta corriente (ahorro externo). D/Y: deuda pública/рів.

Fuente: imf World Economic Outlook Database.

E1 balance de la austeridad es evidente. Las gráficas 4 y 5 muestran los saldos sectoriales en Grecia y España (Portugal, Irlanda e Italia muestran los mismos patrones). Se basan en simple contabilidad macroeconómica. E1 pIB es igual a consumo más inversión más gasto público más saldo de la cuenta corriente de la balanza de pagos. El ahorro privado neto (ahorro menos inversión), el ahorro público (el saldo neto del gobierno) y el ahorro externo (déficit de cuenta corriente) suman cero. En todos los casos mostramos los tres saldos y la relación deuda pública/рıв. Se aprecia claramente que el ajuste fiscal (la tendencia creciente de los ingresos menos los gastos públicos) corrige el déficit de la cuenta externa, mientras que la relación deuda pública/PIB aumenta a lo largo del periodo. La austeridad tiene la finalidad de corregir los desequilibrios externos para mantener bajo control la sostenibilidad de la deuda con el sistema financiero del centro; la deuda pública resulta negativamente afectada, lo que es coherente con el impacto multiplicador de la austeridad sobre el PIB. El objetivo explícito fracasa, el implícito se cumple. 


\section{Gráfica 5}

Saldos sectoriales y deuda pública: España 2007-2013

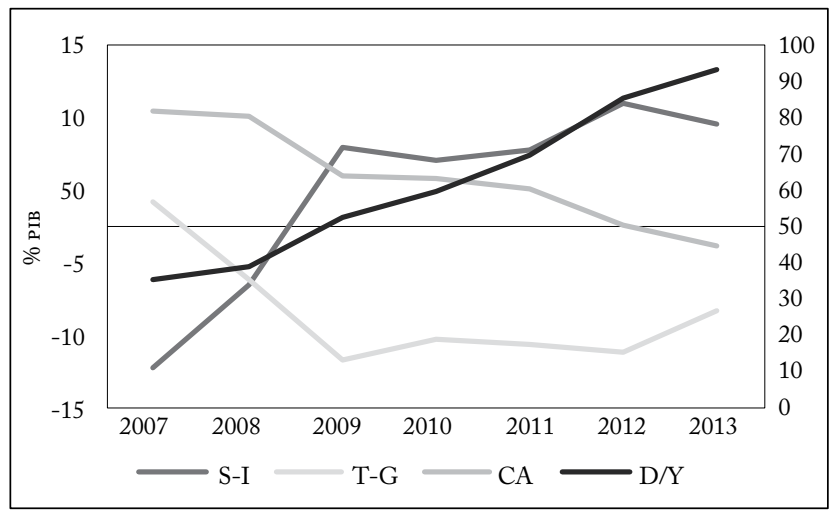

S-I: ahorro neto privado, T-G: ahorro público neto, CA: déficit en cuenta corriente (ahorro externo). D/Y: deuda pública/PiB.

Fuente: IMF World Economic Outlook Database

Las consecuencias de la austeridad son llamativas a nivel comparativo. Estados Unidos, favorecido por una política monetaria acomodadora ${ }^{12}$ y una política fiscal expansiva, registra en 2013 un crecimiento del $6 \%$ respecto al periodo pre-crisis, mientras que la Unión Europea en 2013 tiene un nivel de PІв 1,1\% menor que el nivel pre-crisis. Obviamente hay datos positivos dentro del área, como Alemania, Austria o Francia, pero observamos datos catastróficos para España, Italia, Grecia e Irlanda (cuadro 2).

${ }^{12}$ Una crítica que se hace a menudo a las autoridades europeas es el retraso en la implementación de políticas monetarias expansivas (por ejemplo, la relajación cuantitativa, que llegó mucho después). El retraso es un hecho, pero no hay que interpretarlo como un simple error de política económica, sino que refleja los equilibrios de poder en Europa y en particular la oposición alemana. Es bueno recordar, por ejemplo, que en 2008, cuando el colapso mundial ya era evidente, el все subió las tasas por miedo al precio de petróleo, y en 2011, con Europa hundida, hubo otra subida porque Alemania necesitaba mandar una señal de moderación en el medio de una negociación contractual donde el sindicato IgMetal pedía aumentos salariales elevados (Cesaratto, 2016). Huelga recordar de todos modos que sin política fiscal expansiva una salida de la recesión es imposible por la existencia del límite natural a la política monetaria (el llamado Zero Lower Bound). 
Cuadro 2

Índice del PIB, países seleccionados $(2008=100)$

\begin{tabular}{lcc}
\hline País & PIB 2013 & Variación del PIB 2008-2013\% \\
\hline Alemania & 103,1 & 3,1 \\
Austria & 102,3 & 2,3 \\
Países Bajos & 97,4 & $-2,6$ \\
Polonia & 113,7 & 13,7 \\
Irlanda & 93,3 & $-6,7$ \\
Dinamarca & 97,4 & $-2,6$ \\
Finlandia & 94,1 & $-5,9$ \\
Suecia & 106,3 & 6,3 \\
Francia & 102,3 & 2,3 \\
Reino Unido & 99,2 & $-0,8$ \\
Italia & 92,9 & $-7,1$ \\
Portugal & 93,2 & $-6,8$ \\
España & 92,7 & $-7,3$ \\
Grecia & 76,7 & $-23,3$ \\
Estados Unidos & 106,0 & 6,0 \\
UE (27) & 98,9 & $-1,1$ \\
\hline
\end{tabular}

Fuente: Eurostat, elaboración de los autores.

Además, la crisis lleva a una contracción asimétrica de la manufactura europea: La red productiva alemana ha crecido en tamaño y su capacidad competitiva ha mostrado resiliencia. Al contrario, los países mediterráneos han perdido capacidad productiva y no han sido capaces de recuperarse del impacto negativo de la crisis (cuadro 3). En resumen, la austeridad ha exacerbado la polarización que caracterizaba el área desde antes de la crisis (Simonazzi et al., 2013; Landesmann, 2015; Cirillo y Guarascio, 2015).

La contracción de la base productiva manufacturera tiene consecuencias a largo plazo. En primer lugar, esta es el instrumento para sacar provecho de las oportunidades tecnológicas en los mercados mundiales (Rodrik, 2012; Stöllinger et al., 2013; European Commission, 2013; 2014). En segundo lugar, este sector produce bienes de capital y maquinaria que juegan un papel clave en los servicios esenciales y permiten el enlace con las cadenas globales de valor y con los derrames tecnológicos. Esta destrucción implica por lo tanto una amenaza para la Unión, que pierde terreno respecto a competidores internacionales (Cirillo y Guarascio, 2015) ${ }^{13}$.

${ }^{13}$ Es importante subrayar que la austeridad golpeó, también, la I y D, acentuando la polarización en esta dimensión. Países como Italia y España acentúan su retraso estructural, mientras Alemania y Estados Unidos se consolidan como líderes. A largo plazo, existe evidentemente una conexión entre capacidad competitiva, capacidad productiva manufacturera e inversión en I y D (Cirillo y Guarascio, 2015). 
Cuadro 3

Producto manufacturero/ PIB y variación real del producto manufacturero 2008-2013

\begin{tabular}{|c|c|c|c|}
\hline \multirow{2}{*}{ País } & \multicolumn{2}{|c|}{ Producto manufacturero/PIB } & \multirow[t]{2}{*}{ Variación } \\
\hline & 2008 & 2013 & \\
\hline Alemania & 22,6 & 23,0 & 4,0 \\
\hline Austria & 21,0 & 21,0 & 1,0 \\
\hline Países Bajos & 13,6 & 13,5 & $-2,0$ \\
\hline Reino Unido & 11,2 & 10,5 & $-11,1$ \\
\hline Polonia & 22,1 & 23,6 & 3,7 \\
\hline Francia & 12,8 & 12,5 & 0,3 \\
\hline Italia & 17,4 & 16,2 & $-13,5$ \\
\hline España & 14,6 & 13,1 & $-16,1$ \\
\hline Grecia & 8,3 & 7,2 & $-34,3$ \\
\hline UE 27 & 16,7 & 16,3 & $-2,9$ \\
\hline
\end{tabular}

Fuente: Eurostat, elaboración de los autores.

\section{LA CRISIS GRIEGA}

$E x$ post, la historia del rescate griego fue ejemplar, por ser Grecia el país más débil de la periferia, así como el más beneficiado por la entrada de capitales, y por ser el caso donde más se evidencia el entrelazamiento de la visión idealista-política e ideológico-económica. Seis años después del primer paquete de ajustes, el PIB griego se ha reducido un 20\% (gráfica 6), el costo del trabajo se ha reducido, en términos nominales, un 18\% (gráfica 7). El tamaño de un ajuste similar solo se documentó en Europa en un periodo de guerra.

Los paquetes de ajuste condicional llegaron endulzados por previsiones de la troika (FMI-BCE-Comisión Europea) sobre la recuperación de la economía. Estas se muestran en Darvas (2015) usando los datos Ameco: a medida que el tiempo pasa, se sigue pronosticando un rebote en el PIв que no se materializa, con una distancia entre lo pronosticado y lo ocurrido que sobrepasa cualquier error estadístico (alrededor del 20\%). Tomando el pIB real en 2009 como base, la primera previsión señalaba una reversión en 2011, con una recuperación casi completa en 2014; la primera corrección, que a su vez es un segundo escenario, reconoce que la ruptura del patrón no se materializa, y prevé una estabilización en 2012 y un rebote que llevaría al рів de 2014 a un nivel 12\% más bajo que el de 2009. En realidad, de acuerdo a la senda actual, el PIB en 2014 era más de 20 puntos más bajo que en 2009. Ex post, el mismo fondo admitió su impreparación, y reconoció que las decisiones adoptadas y las previsiones formuladas respondieron más a lógicas de group thinking, que al análisis económico (IEO, 2016). 
Gráfica 6

Índice del рів $(2010=100)$

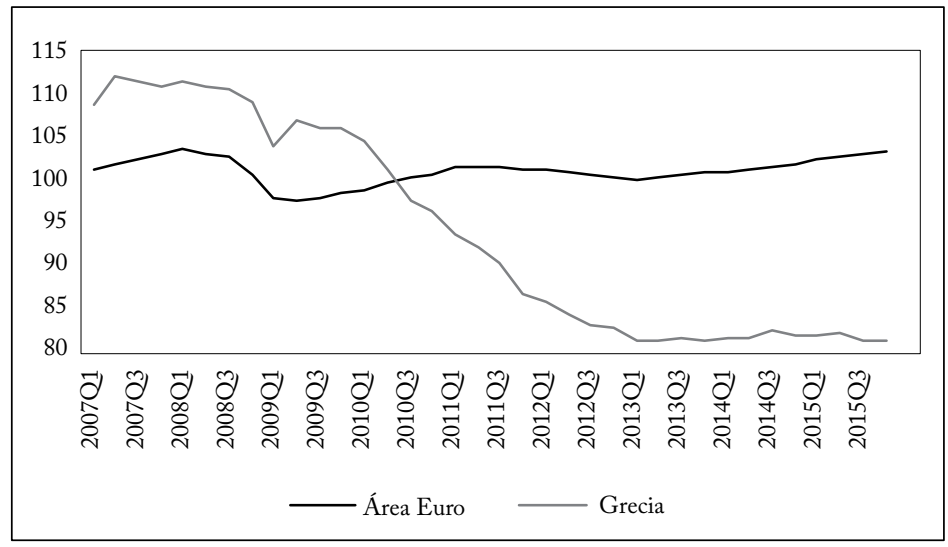

Fuente: Eurostat.

Gráfica 7

Índice del costo laboral para Grecia, en términos nominales



Fuente: Eurostat.

Usando datos del Bank for International Settlements (gráfica 8) se puede ver cómo el dinero prestado a Grecia fue en realidad un rescate de bancos alemanes y franceses. Al final de 2012, la exposición privada se vuelve exigua; la deuda griega se ha transformado en préstamos emitidos bajo leyes internacionales, protegiendo a los acreedores ante el riesgo de devaluación y frenando la posibilidad de un plan $\mathrm{B}$ para Grecia (la salida de la moneda común).

Sin embargo, la lección relevante del caso griego es el funcionamiento del piloto automático. 
Grecia representa alrededor del 2\% del PIв del área euro, con una deuda pública que en el momento de la crisis era cercana al 100\% del PIв. Obviamente, el sistema financiero habría sido capaz de aguantar hasta una cancelación total de la deuda, con el sostén de los organismos internacionales. La atención era captada, en ese momento, por el riesgo de contagio, ya que el precedente de Lehman Brothers había mostrado que un solo episodio de quiebra de magnitud relativamente contenida sería capaz de vaciar el mercado interbancario. El euro es un acuerdo de cambio sui generis, ya que prevé la adopción de una nueva moneda y no formula un procedimiento de salida en sus tratados de fundación (como por ejemplo ocurre con la Unión Europa, donde hay un procedimiento reglamentado por el artículo 50). En teoría económica se dice que puede funcionar solo si es renegotiation proof (Hart y Moore, 1988).

Gráfica 8

Exposición consolidada Bancos hacia Grecia



Fuente: Bank for International Settlements.

Grecia tiene una estructura económica atrasada respecto tanto al centro como a la periferia: sectorialmente, se caracteriza por el papel clave de una oligarquía relacionada con el sector portuario (reexportación de mercancías) y financiero, mientras que la gran parte de la clase media está involucrada en actividades como el turismo, que tiene un carácter casi informal, estando prácticamente ausente un sector industrial. Esto impone cierto tipo de restricción sobre el país, que por ejemplo depende mucho de las importaciones y tiene elasticidades externas comercio-precios muy bajas, por lo que en- 
frenta muchas dificultades ante una devaluación y, por ende, frente al escenario de una salida de la moneda común (Papadimitriou et al., 2014) ${ }^{14}$.

Adicionalmente, Grecia había logrado entrar en el euro a través de una serie de manipulaciones contables, para mover rubros de balance (Kouvelakis, 2011) ${ }^{15}$. Este hecho es reprochado a lo largo de toda la crisis griega, con tonos de regaño y matices religiosos. La manipulación contable pudo ser de un $0,7 \%$ a un $1 \%$ del PIB (oECD, 2005), mientras que el ajuste al cual fue sometido el país fue de aproximadamente 20 puntos del PIB.

Los vaivenes de los paquetes de ajuste y de los gobiernos (elegidos, técnicos, de coalición) son acompañados por protestas masivas, que ganan apoyo en gran parte de los movimientos anti-austeridad. En ese clima nace Syriza, una coalición de movimientos y partidos que se juntan alrededor de Synaspismos, la descendiente del partido comunista filo-europeo (y distinto del кKE que es el tradicional Partido Comunista Griego, con posiciones anti-europeas). No existe un verdadero programa, más allá de un vago llamado a Nicos Poulantzas (1977; 1979). Las posiciones van desde una clara oposición a la UE y al euro (Lapavitsas, 2012), hasta posiciones más filo-europeas, como las de Alexis Tsipras.

En las elecciones legislativas de 2012 Syriza termina como segundo partido, empezando a perfilarse como una opción real de gobierno, e intenta transformar el partido siguiendo dos líneas claras: la construcción de un proceso unitario, alrededor del liderazgo de Tspiras, y la suavización de la línea radical, con una postura anti-austeridad pero rígidamente dentro de un cuadro filo-europeo (Kouvelakis, 2016). Hay muchas razones detrás de estas decisiones, también

\footnotetext{
${ }^{14}$ Grecia se transformó en una social-democracia en los años ochenta, cuando ya la mayoría de los países europeos enfrentaban un viraje en la política económica de tipo liberal; una transformación guiada por el PAsoK (el partido socialista) con una expansión significativa del sector público. E1 mismo PAsoK guio la transformación en sentido (neo)liberal en los años 1996-2004, que llevan a la meta de la entrada en la moneda común (Kouvelakis, 2011). Estas múltiples transformaciones no alteran un contrato social basado en un sistema de recaudo muy atrasado, de manera que, si bien en magnitud diferente, tanto los ricos como la clase media-baja se benefician de la evasión tributaria.

${ }_{15}$ Nos referimos al cambio de criterios contables que ocurrió entre 1997 y 1999, que llevó en 2002 a que Eurostat rechazara los números del gobierno griego. Se suele confundir este episodio con la firma de un contrato de swap con Goldman Sachs que permitió esconder una parte de la deuda para transferirla a futuro. Este último episodio ocurrió en 2002, con el país ya miembro del euro. Este contrato generó bastante indignación en la opinión pública ya que entre los responsables del banco de inversión para Europa estaban algunos de los miembros de la tecnocracia que luego impulsó las políticas de austeridad en el país heleno.
} 
de cálculo político, ya que la fascinación por el euro en la opinión pública griega $-\mathrm{y}$ española y portuguesa- era alta, probablemente porque se veía como el final de un camino de desarrollo económico y social que llevó a estos países de las dictaduras hacia el club de las social-democracias.

Ex post, la equivocación fue clara: la bandera anti-austeridad se sustentaba en la visión programática de una alternativa de política económica, dentro del esquema euro, sin poner en discusión las restricciones institucionales que determinan, como mostramos, la presión a intervenciones pro-cíclicas. El programa se centraba en el mantra de resolver la emergencia humanitaria, buscando una forma de negociación con las autoridades europeas (Kouvelakis, 2016).

La estrategia paga electoralmente ya que Syriza gana las elecciones de 2015, pero los acontecimientos de ese mismo año revelan cómo el choque entre instituciones y policies se resuelve a favor de las primeras, lo que es coherente con el esquema del piloto automático.

Tsipras usa una estrategia de diálogo. No promueve controles de capitales, debilitando su margen de acción ya que la fuga de capitales deja la ELA como única válvula de oxígeno para el sistema bancario.

Esa situación de buscar un margen dentro del conjunto de opciones (escasas y limitadas) que manejan Alemania y las instituciones europeas es evidentemente infecunda, y Europa vive un estancamiento de la situación hasta julio, cuando se da el golpe de escena más fuerte. En ese mes, Tspiras llama a un referéndum sobre el Memorándum que Europa le propone al país helénico. En Europa todas las oposiciones arropan a Syriza en su batalla por el oxi ("no" en griego), que gana ampliamente, a pesar de campañas mediáticas muy alarmistas (Bogliacino et al., 2015).

Sin embargo, no hay margen de acción: el combinado institucional está diseñado para resistir a las presiones que puedan llegar desde abajo. El mismo Tsipras anuncia, a una semana del "No", que firmará un acuerdo. Las condiciones son durísimas: reforma de las pensiones con subida de la edad de jubilación y recorte a las pensiones mínimas; privatización por 52 mil millones de euros; compromiso a reformar el mercado del trabajo, obviamente todo bajo el control estricto de la troika (Guarascio y Bogliacino, 2015). Sencillamente, el espacio político no existía. Obviamente, la deteriorada situación económica y la presente estagnación de la economía hacen que el riesgo de una salida no esté descartado. 


\section{LA DISFUNCIONALIDAD DE LAS INSTITUCIONES POS CRISIS}

Primero la Unión Monetaria Europa (los acuerdos de Maastricht de 1992) y luego los acuerdos institucionales firmados después de la Gran Recesión ${ }^{16}$ institucionalizan la austeridad como respuesta correctiva a cualquier choque.

A nivel europeo, entran en vigor las legislaciones llamadas Six Pack (European Parliament, Council of the European Union 2011a; 2011b; 2011c; 2011d; Council of the European Union 2011a; 2011b), Treaty on Stability, Coordination and Governance (TscG) y Two Pack (European Parliament, Council of the European Union 2013a; 2013b) ${ }^{17}$. El Six Pack reafirma las reglas de mantener un objetivo a largo plazo del 3\% del рів para el déficit público y del 60\% del PIв para la deuda pública. E1 Two Pack establece los mecanismos de monitoreo y vigilancia para los países miembros y sobre todo para los países con exceso de déficit y para los países en situación de fragilidad financiera. E1 Tratado incluye una parte fiscal que ha sido bautizada Fiscal Compact. Este último impone un límite del 0,5\% para el déficit estructural (1\% si la relación deuda pública/ Рів del país es inferior al $60 \%)$ y requiere de mecanismos de corrección automática para converger hacia los objetivos de largo plazo antes mencionados. Como detalle fundamental, el Tratado exige una implementación a través de "disposiciones que tengan fuerza vinculante y sean de carácter permanente, preferentemente de rango constitucional”. Como explica Giacché (2015), una vez introducidas en las constituciones nacionales se vuelven un monstruo jurídico que supedita el poder constitucional y la amplitud de derechos económicos y sociales que se pueden legitimar y garantizar democráticamente dentro del contrato social.

Si recordamos la prescripción de la teoría, se necesitaría un mecanismo de transferencia y coordinación (algo como compartir el

${ }^{16}$ El objetivo y el valor añadido de una moneda común debería ser la creación de un mercado interno más amplio (Alesina et al., 2002). Esto cumpliría la función de suavizar el impacto de choques externos. Sin embargo, como recuerda Bagnai (2012b), si los países son homogéneos, las necesidades de ajustes del tipo de cambio y los costos de transacción son mínimos. Esto equivale a decir que el acuerdo es necesario allí donde no se debería hacer. Por otro lado, el problema dentro de áreas heterogéneas es que las asimetrías entre deudores y acreedores generan presiones deflacionarias: el que tiene saldo externo positivo no tiene ninguna necesidad de ajustar, mientras que, por el contrario, el deudor tiene que adoptar medidas de balance para corregir la cuenta externa. La austeridad descrita anteriormente, como mostramos, tiene un impacto procíclico, destruyendo la demanda interna y reduciendo el mercado común, precisamente lo contrario del objetivo inicial de la moneda común.

${ }^{17}$ El estatus de cada una es diferente: el Six Pack está compuesto por cinco regulaciones y una directiva, el Tratado es un acuerdo intergubernamental, y el Trwo Pack está compuesto por dos regulaciones. 
riesgo). Por ejemplo: ante una crisis en España, el mecanismo debe reducir allí el recaudo de impuestos y aumentarlo en Alemania, de modo que el impacto en el gasto sea opuesto en los dos países. Las medidas de control y de supervisión deberían como mínimo ser acompañadas por un mecanismo de este tipo. Sin embargo, como afirma Bagnai (2012b), esto no es políticamente viable, ya que las opiniones públicas del centro no lo aceptarían nunca, después de ser exaltadas con declaraciones en contra de los países en deuda (p. ej., la negativa a los Eurobond de Merkel fue rotunda en las campañas electorales; Ferrera, 2016). Lo más importante es que su implementación tampoco sería deseable: el equilibrio de poder impondría una centralización tecnócratica de las decisiones presupuestales, agravando el actual déficit democrático de vigilancia sobre la política económica (Arestis y Sawyer, 2012).

Así como para las cuentas públicas hay reglas fiscales sin mutualizar la deuda (Eurobond), en el sistema bancario hay unión bancaria con bail in $^{18}$, pero sin garantía general de los depósitos.

Los elementos clave de la Unión Bancaria son tres: reglas comunes de carácter prudencial (conforme a las reglas de Basilea), supervisión única de los bancos bajo el mando del вCE, y un mecanismo de resolución para bancos en quiebra (European Parliament, Council of the European Union, 2014; 2013c; 2013d; Council of the European Union 2013a; 2013b). Estos mecanismos eliminan la fragmentación del mercado común, algo favorable a los grupos bancarios por las economías de escala, pero generan efectos perversos, ya que agravan el fenómeno "demasiado grande para quebrar". De hecho, en presencia de quiebra, la única salida posible es la incorporación por otro grupo, lo que aumenta la concentración.

Adicionalmente, el mecanismo único de resolución financiado por el sistema financiero es pequeño para cubrir una quiebra grande, que si llegara a ocurrir abriría la puerta a la intervención con dinero de los contribuyentes y con el tradicional paquete de ajuste condicional, e intervendría solo después de recurrir a los fondos del banco, incluyendo la cláusula de bail in. Bajo esta última, tanto los titulares de cuentas de ahorros como los que subscribieron obligaciones comparten el riesgo por encima de un umbral.

\footnotetext{
${ }^{18}$ Participación de obligacionistas y hasta de titulares de cuentas de ahorros por encima de un umbral en caso de quiebre y rescate. E1 deterioro de los balances de los bancos italianos, determinado también por la crisis de confianza que sigue a la introducción del bail in, y obviamente por las políticas de austeridad, puede llevar a una nueva crisis financiera. Esto y otros sucesos políticos inminentes, como varias elecciones en países clave, permite afirmar que el riesgo de quiebra del euro no está eliminado todavía.
} 


\section{CONCLUSIONES}

Este artículo se concentra en los factores estructurales e institucionales que llevaron a la crisis del euro. En primer lugar, se muestra que el proceso de integración monetaria fue influenciado por dos cambios fundamentales: 1) el paso a un régimen de acumulación caracterizado por el libre movimiento de capitales, la expansión del sector financiero, el crecimiento del papel de las exportaciones y el incremento de la cuota de capital en la distribución; 2) el desbalance económico hacia el centro por la reunificación alemana (concretamente, Alemania y su red productiva y el eje franco-alemán en términos de concentración financiera).

En segundo lugar, se muestra que la construcción de la UME engendra las condiciones para un proceso de divergencia y de inestabilidad financiera, de acuerdo con el clásico ciclo financiero. La introducción del euro abre el desequilibrio interno al área y siembra las semillas de la crisis, que se manifiesta con la primera perturbación externa. En ausencia de un mecanismo de reequilibrio, la respuesta es la imposición de una consolidación fiscal en la periferia, la llamada austeridad. Esta última arregla las cuentas externas destruyendo demanda interna, pero de esta manera complica las perspectivas económicas de las familias y las empresas, empeorando sus balances y perjudicando la posibilidad de pagar sus deudas. Como resultado, se deteriora la situación patrimonial de los bancos, que están expuestos a una nueva crisis financiera. Obviamente, el ciclo financiero tiene una componente política clave: frente a una crisis, la UE no ofrece un mecanismo de salvaguardia, sino que ofrece un mecanismo de rescate, bajo condiciones que fijan los capitales del centro y sin supervisión de mecanismos democráticos, y ofrece el bail in, que al mismo tiempo golpea los ahorros de personas que legítimamente pueden no ser conscientes del perfil de riesgo de sus inversiones. Al no presentar una solución definitiva al problema (por la ausencia de la implementación de la garantía común) se puede generar pánico y una nueva crisis generalizada.

Ese núcleo contradictorio a nivel estructural e institucional ha sido arropado por dos lecturas políticas dominantes que sustancian el discurso político europeo. La combinación de las dos visiones conduce a una situación de inefectividad que está llevando a un proceso de disgregación dominado por la incertidumbre (Bassi et al., 2016).

La primera visión, que hemos definido ideológico-económica y que se puede identificar con impostaciones ortodoxas propuestas entre otros por Sinn (2014), postula una sustancial dominancia de 
las fuerzas económicas en un proceso donde se excluyen a priori las fuentes de crisis. Es una visión de libro de texto, que ha llevado a creer que la eliminación de las tasas de cambios conduciría a una rápida convergencia de las economías más atrasadas hacia las más desarrolladas (Alemania en este caso). Una convergencia impulsada por los flujos de capitales desde el centro hacia la periferia, supuestamente instrumentales para un crecimiento de la productividad. En este marco, son necesarias las reformas por el lado de la oferta (recortar salarios directa o indirectamente, por ejemplo debilitando el poder contractual de los trabajadores), para así garantizar competitividad de precios, pero descuidando el rol de la calidad y la tecnología en la producción (Storm y Naastepad, 2015).

Los datos nos muestran que sí hubo una entrada masiva de capitales, pero que no hubo un despegue de la productividad sino burbujas, que, alimentando procesos inflacionarios, profundizaron los desequilibrios externos y debilitaron las economías de la periferia. Las soluciones -elaboradas dentro de la misma visión- tampoco sirvieron, ya que la austeridad, que corrigió el déficit externo destruyendo la demanda interna, tuvo efectos a largo plazo sobre la capacidad de pago de la deuda y condujo a un empeoramiento de los balances bancarios, amenazando con una nueva crisis.

La segunda visión es igualmente maniquea, pero es unidimensionalmente política (Negri y Sánchez, 2015). Esta visión interpreta el proceso de integración como la simple postulación de un ideal federalista europeo (el mencionado Manifiesto de Ventotene), más allá de la complejidad epistémica de la realidad de los países miembros. Esta visión se reforzó en los años noventa, con la aceleración del proceso de integración, incurriendo en múltiples deslices semánticos, como la confusión entre Europa y euro, entre otros. Esta ambivalencia representa un potente antídoto contra las críticas, que, por ejemplo, intenten distinguir los fines de una real convergencia económica de las economías europeas de los medios de la actual infraestructura institucional. La deseabilidad de los primeros no legitima como eficaz o eficiente la segunda.

El entrelazamiento de las dos perspectivas ha sido fatal para el proceso europeo. La perspectiva económica que postula el desarrollo armónico produce como reacción natural instituciones esquizof rénicas que, dado su mal funcionamiento, perjudican la posibilidad de alcanzar la meta. El discurso político de la segunda visión legitima la construcción institucional bajo la perspectiva de un cambio de políticas, pero termina sacrificándose en su propio altar, ya que las 
restricciones institucionales eliminan el margen de construcción de una alternativa política.

La lección griega, finalmente, representa un momento cumbre para entender esta interacción entre la dimensión económico-ideológica y la dimensión político-idealista. Primero se desatan esperanzas referidas a una alternativa de integración y posteriormente se da un proceso de alineación impulsado por los vínculos institucionales ( $l a$ pistola apuntando), que termina siendo un instrumento de legitimación del proceso en curso.

Con el continente en medio de una década perdida y sufriendo tensiones que amenazan con disgregarlo, el dispositivo mencionado termina siendo la clave para entender cómo se alimenta la actual parálisis política y la esterilidad de alternativas.

\section{REFERENCIAS BIBLIOGRÁFICAS}

1. Alesina, A. y S. Ardagna. "Large changes in fiscal policy: Taxes versus spending" [2009], J. R. Brown, ed., Tax policy and the economy 24, 2010, pp. 35-68.

2. Alesina, A.; R. J. Barro y S. Tenreyo. "Optimal currency areas", M. Gertler y K. Rogoff, eds., nber Macroeconomics Annual 17, 2002, pp. 301-356.

3. D'Angelillo, M. y L. Paggi. "Deutschland, Deutschland... Über A1les”, A. Cesaratto y M. Pivetti, eds., Oltre l'austerità, Gli eBook di MicroMega, 2012, pp. 61-78.

4. Ardagna, S. y F. Caselli. "The political economy of the Greek debt crisis: A tale of two bailouts", Centre for Economic Performance special paper 25, Londres, 2012.

5. Arestis, P. y M. Sawyer. "The dangers of pseudo fiscal inion in the emu”, 2012, [http://triplecrisis.com/the-dangers-of-pseudo-fiscalunion-in-the-emu/].

6. Atkinson, A. B. Inequality. What can be done?, Cambridge, Mass., Harvard University Press, 2015.

7. Bagnai, A. Il tramonto dell'euro, Reggio Emilia, Imprimatur, 2012a.

8. Bagnai, A. "Le aporie del più Europa", S. Cesaratto y M. Pivetti, eds., Oltre l'austerità, Gli eBook di MicroMega, 2012b, pp. 49-60.

9. Barba, A. y M. Pivetti. La scomparsa della sinistra in Europa, Reggio Emilia, Imprimatur, 2016.

10. Bassi, F.; F. Bogliacino et al. "Brexit: The next domino could be Italy. Social Europe", 2016, [https://www.socialeurope.eu/2016/07/ brexit-the-day-after/ ].

11. Blanchard, O. y D. Leigh. "Growth forecast errors and fiscal multipliers”, IMF working paper WP/13/1, 2013.

12. Bogliacino, F. y V. Maestri. "Growing income inequalities?", W. Salverda, B. Nolan et al., eds., Changing inequalities and societal impacts in rich countries: Analytical and comparative perspectives, Oxford, Oxford University Press, 2014. 
13. Bogliacino, F.; D. Guarascio y V. Cirillo. "Una lezione di democrazia”, Il Manifesto, 30/06/2015, [http://ilmanifesto.info/una-lezionedi-democrazia/].

14. Bogliacino, F.; D. Guarascio y V. Cirillo. "The dynamics of profits and wages: Technology, offshoring and demand", LEM Papers Series 2016/04, Sant'Anna School of Advanced Studies, Pisa, 2016.

15. Cafruny, A. W. "European integration studies, European Monetary Union, and resilience of austerity in Europe: Post-mortem on a crisis foretold", Competition E Change 19, 2, 2015, pp. 161-177.

16. Celi, G.; A. Ginzburg et al., Northern and Southern Europe in the long European Crisis: a core-periphery perspective, Londres, Routledge, de próxima publicación, 2017.

17. Cesaratto, S. Sei lezioni di economia, Reggio Emilia, Imprimatur, 2016.

18. Cirillo, V. y D. Guarascio. "Jobs and competitiveness in a polarised Europe", Intereconomics 50, 3, 2016, pp. 156-160.

19. Constâncio, V. "The European crisis and the role of the financial system", discurso del vice presidente del все en Atenas, 23 de mayo de 2013, [www.ecb.europa.eu/press/key/date/2013/html/sp130523_1. en.html].

20. Council of the European Union. "Regulation (EU) No 1177/2011", 8 de noviembre de 2011a, [www.eur-lex.europa.eu/legal-content/Es/ $\mathrm{TXT} /$ ?uri=CELEX\%3A32011R1177].

21. Council of the European Union. "Council Directive 2011/85/EU", 8 de noviembre de 2011b, [www.eur-lex.europa.eu/legal-content/EN/ ALL/?uri=uriserv:OJ.L_.2011.306.01.0041.01.ENG].

22. Council of the European Union. "Council Regulation (EU) No 1024/2013”, 15 de octubre de 2013a, [www.eur-lex.europa.eu/legalcontent/EN/TXT/?uri=CELEX:32013R1024].

23. Council of the European Union. "Regulation (EU) No 1022/2013", 22 de octubre de 2013b, [www.eur-lex.europa.eu/legal-content/EN/ TXT/?uri=CELEX:32013R1022].

24. Darvas, Z. “Is Greece destined to grow?", Bruegel post, 2015, [http:// bruegel.org/2015/06/is-greece-destined-to-grow/].

25. De Leo, M. “Spread: l'educazione dei greci”, S. Cesaratto y M. Pivetti, eds., Oltre l'austerità, Gli eBook di MicroMega, 2012, pp. 178-191.

26. De Long, J. B.; A. Shleifer et al. "Noise trader risk in financial markets", Journal of Political Economy 98, 1990, pp. 703-738.

27. Díaz-A., C. "Good-bye financial repression, hello financial crash", Journal of Development Economics 19, 1-2, 1985, pp. 1-24.

28. European Commission. "Towards knowledge driven reindustrialization”, European competitiveness report final, Bruselas, 2013.

29. European Commission. "Helping firms grow", European competitiveness final report, Bruselas, 2014.

30. European Parliament. "Regulation (Eu) No 806/2014 of the European Parliament and of the Council”, 15 de julio de 2014, [www.eur-lex. europa.eu/legal-content/en/TXT/?uri=celex\%3A32014R0806].

31. European Parliament. "Regulation (EU) No 473/2013 of the European Parliament and of the Council”, 21 de mayo de 2013a”, [www.eurlex.europa.eu/legal-content/EN/ALL/?uri=CELEX\%3A32013R0473]. 
32. European Parliament. "Regulation (EU) No 472/2013 of the European Parliament and of the Council", 21 de mayo de 2013b, [www. eur-lex.europa.eu/legal-content/EN/ALL/?uri=celex\%3A32013R0472].

33. European Parliament. "Regulation (EU) No 575/2013 of the European Parliament and of the Council", 26 de junio de 2013c, [www.lex. europa.eu/legal-content/EN/TXT/?uri=OJ:L:2013:176:TOC].

34. European Parliament. "Directive 2013/36/EU of the European Parliament and of the Council", 26 de junio de 2013d, [www.eur-lex. europa.eu/legal-content/EN/TXT/?uri=OJ:L:2013:176:TOC].

35. European Parliament. "Regulation (EU) No 1173/2011 of the European Parliament and of the Council", 16 de noviembre de 2011a, [www. eur-lex.europa.eu/legal-content/EN/ALL/?uri=CELEX:32011R1173].

36. European Parliament. "Regulation No. 1174/2011 of the European Parliament and of the Council, 16 de noviembre de 2011b, [www.eurlex.europa.eu/legal-content/EN/ALL/?uri=CELEX\%3A32011R1174].

37. European Parliamen. "Regulation (EU) No. 1175/2011 of the European Parliament and of the Council", 16 de noviembre de 2011c, [www.eurlex.europa.eu/legal-content/EN/ALL/?uri=CELEX\%3A32011R1175].

38. European Parliament. "Regulation 1176/2011 of the European Parliament and of the Council", 16 de noviembre de 2011d, [www. ex.europa.eu/legal-content/en/TXT/?uri=celex\%3A32011R1176].

39. Ferrera, M. Rotta di collisione. Euro contro welfare?, Bari, Laterza, 2016.

40. Fisher, F. "Disequilibrium foundations of equilibrium economics", Econometric society monographs, 1983.

41. Flassbeck H. y C. Lapavitsas. Against the troika: Crisis and austerity in the eurozone, Londres y Nueva York, Verso, 2015.

42. Foucault, M. L'ordre du discours, Paris, Gallimard, 1970.

43. Frankel, J. A. y A. K. Rose. "Is EMU more justifiable ex post than ex ante?", European Economic Review 41, 3-5, 1997, pp. 753-760.

44. Frenkel, R. "What have the crises in emerging markets and the Euro Zone in common and what differentiates them?”, J. E. Stiglitz y D. Heymann, eds, Life after debt, International Economic Association, 2014, pp. 122-141.

45. Giacché, V. Costituzione italiana contro trattati europei. Il conflitto inevitabile, Reggio Emilia, Imprimatur, 2015.

46. Guarascio, D. y F. Bogliacino. "Europa anno zero”, A. Mastrandrea, ed., Grecia-Europa, cambiare è possibile? Cinque anni tra crisi e alternative, 2015, [http://sbilanciamoci.info/wp-content/uploads/2012/04/ Sbilibro12_Grecia-Europa_web.pdf].

47. Hart, O. y J. Moore. "Incomplete contracts and renegotiation", Econometrica 56, 4, 1988, pp. 755-785.

48. Herndon, T.; M. Ash y R. Pollin. "Does high public debt consistently stifle economic growth? A critique of Reinhart and Rogoff", Cambridge Journal of Economics 38, 2, 2014, pp. 257-279.

49. Higgins, M. y T. Klitgaard. "The balance of payments crisis in the Euro Area periphery. Current issues in Economics y Finance", Federal Reserve Bank of New York 20, 2, 2014, pp. 1-8. 
50. Hope, K. “Greek central bank accused on short selling”, Financial Times, 29 de mayo de 2010, [http://www.ft.com/intl/cms/s/0/28afd82a8ea1-11df-8a67-00144feab49a.html\#axzz46C6nwAuc].

51. Iеo. "The imf and the Crises in Greece, Ireland, and Portugal: An evaluation by the Independent Evaluation Office", 2016, [http://www. ieo-imf.org/ieo/files/completedevaluations/EAC__REPORT\%20v5.PDF ].

52. Jonung, L. y E. Drea. "It can't happen, it's a bad idea, it won't last: us economists on the EMU and the Euro, 1989-2002", Economic Journal Watch 7, 1, 2010, pp. 4-52.

53. Kaminska, I. "The Romans always copy the Greeks (including the repo market)", Financial Times, 20 de Julio de 2011, [http://ftalphaville.ft.com/2011/07/20/627896/the-romans-always-copy-the-greeksincluding-the-repo-market/].

54. Keynes, J. M. The general theory of employment, interest and money, Londres, Macmillan, 1936.

55. Kouvelakis, S. "Syriza's rise and fall”, New Left Review 97, 2016, pp. 45-70.

56. Kouvelakis, S. “The Greek cauldron”, New Left Review 72, 2011, pp. 17-32.

57. Krugman, P. “The euro: Beware of what you wish for”, Fortune, diciembre de 1998, [http://web.mit.edu/krugman/www/euronote.html].

58. Landesmann, M. "The new north-south divide in Europe: Can the European convergence model be resuscitated?”, Farberger, J. et al., eds., The triple challenge for Europe: Economic development, climate change, and governance, Nueva York, Oxford University Press, 2015, pp. 60-89.

59. Lapavitsas, C. Eurocrisis, Londres y Nueva York, Verso, 2012.

60. Minsky, H. P. John Maynard Keynes, Nueva York, Columbia University Press, 1975.

61. Monnet J. Cittadino d'Europa: 75 anni di storia mondiale, Santancargelo di Romagna, Rusconi, 1978.

62. Morris, B. "Greece debt cut: Some of Europe's biggest banks hit", ввс News, 21 de febrero de 2012, [ http://www.bbc.com/news/business-17110355].

63. Mundell, R. A. "A theory of optimum currency areas", American Economic Review 51, 4, 1961, pp. 657-665.

64. Negri, A. y R. Sánchez. "The New Left in Europe needs to be radical - and European", The Guardian, 27 de febrero de 2015, [www. theguardian.com/commentisfree/2015/feb/27/new-left-europe-greecedemocratic-capitalism-nato].

65. O'Connell, A. "European crisis: A new tale of center-periphery relations in the world of financial liberalization/globalization?", International Journal of Political Economy 44, 3, 2015, pp. 174-195.

66. OECD. “Greece 2005”, oECD economic surveys, oECD Publishing, 2005.

67. Papadimitriou, D. B.; M. Nikiforos y G. Zezza. "Prospects and policies for the Greek economy”, Strategic Analysis, Levy Economics Institute, febrero de 2014.

68. Panitch, L. y S. Gindin. The making of global capitalism, Londres y Nueva York, Verso, 2012. 
69. Poulantzas, N. Estado, poder y socialismo, Madrid, Siglo xxi, 1979.

70. Poulantzas, N. Las clases sociales en el capitalismo actual, Madrid, Siglo XXI, 1977.

71. Reinhart, C. M. y K. S. Rogoff. "Growth in a time of debt", American Economic Review, Papers and Proceedings, 100, 2010, pp. 573-578.

72. Rodrik, D. “The manufacturing imperative”, Project-Syndicate, 10 de agosto de 2012, [https://www.project-syndicate.org/commentary/ the-manufacturing-imperative?barrier=accessreg].

73. Simonazzi, A.; A. Ginzburg y G. Nocella. "Economic relations between Germany and Southern Europe", Cambridge Journal of Economics 37, 3, 2013, pp. 653-675.

74. Simonazzi, A. y A. Ginzburg. "The interruption of industrialization in Southern Europe: A center-periphery perspective”, M. Baumeister y R. Sala, eds., Southern Europe? Italy, Spain, Portugal, and Greece from the 1950s until the present day, Frankfurt, Campus, 2015, pp. 103-137.

75. Sinn, H. W. "Austerity, growth and inflation: remarks on the Eurozone's unresolved competitiveness problem", The World Economy 37, 1, 2014, pp. 1-13.

76. Spinelli, A. y E. Rossi. "For a free and United Europe. A draft manifesto", 1941, [http://www.federalists.eu/uef/library/books/theventotene-manifesto/].

77. Stiglitz, J. E. Globalization and its discontents, Nueva York, W.W. Norton, 2002.

78. Stiglitz, J. E. The euro. How a common currency threatens the guture of Europe, Nueva York, W.W. Norton, 2016.

79. Stockhammer, E. "The euro crisis and the contradiction of neoliberalism in Europe”, PKSG working paper 1401, 2014.

80. Stöllinger, R.; N. Foster et al. "A 'manufacturing imperative' in the EU - Europe's position in global manufacturing and the role for industrial policy", wiIw research reports 391, octubre de 2013.

81. Storm, S. y C. W. Naastepad. "Europe's hunger games: Income distribution, cost competitiveness and crisis", Cambridge Journal of Economics 39 3, 2015, pp. 959-986.

82. Van Apeldoorn, B. “The contradictions of 'embedded neoliberalism' and Europe's multi-level legitimacy crisis: The European project and its limits", Contradictions and limits of neoliberal European governance, Chippenham, Palgrave Macmillan, 2009, pp. 21-43. 